\title{
Diseño conceptual de un gasificador de biomasa DE LECHO FIJO EN EQUICORRIENTE A ESCALA PILOTO
}

\author{
Juan Fernando Pérez Bayer ${ }^{1}$ \\ Óscar Homero Díaz Ibarra ${ }^{1}$ \\ Roberto Carlos Obando EnRiqueZ ${ }^{2}$ \\ Alejandro Molina OCHOA ${ }^{3}$
}

\section{Resumen}

En este trabajo se presenta el diseño conceptual de un gasificador de biomasa lignocelulósica de lecho fijo en equicorriente a escala piloto.

La metodología de diseño se basó en el dimensionamiento del reactor a partir de la potencia requerida $(150 \mathrm{kWt})$, y la simulación con un modelo cinético unidimensional del proceso de gasificación. Se buscó obtener rendimientos termodinámicos entre 60 y $75 \%$ para distintos niveles de humedad de biomasa. De acuerdo con la dimensión del reactor y el contenido de humedad de la biomasa, se definieron dos configuraciones óptimas para el gasificador que generan 117 $\mathrm{kWt}$ y $200 \mathrm{kWt}$, con rendimientos termodinámicos del $43 \%$ y $70 \%$, respectivamente. El reactor diseñado tiene 1,8 m de longitud, y produciría entre $25 \mathrm{kWe} \mathrm{y} 50 \mathrm{kWe}$.

El buen rendimiento termodinámico se logró gracias a la adiabaticidad en la zona endotérmica y al aprovechamiento de la entalpía sensible del gas en el secado y la devolatilización de la biomasa.

1 Grupo de manejo eficiente de la energía (GIMEL). Dpto. Ingeniería Mecánica, Facultad de Ingeniería, Universidad de Antioquia, Medellín - Colombia. juanpb@ udea.edu.co

2 Ingeniero Mecánico. Universidad Nacional de Colombia, sede Medellín. Medellín - Colombia.

3 Grupo de bioprocesos y flujos reactivos, Facultad de Minas. Universidad Nacional de Colombia, Sede Medellín. Medellín - Colombia. amolinao@bt.unal.edu.co

Fecha de recepción: 25 de marzo de 2009

Fecha de aceptación: 12 de mayo de 2009 


\section{Palabras clave}

Gasificador en equicorriente, diseño conceptual, biomasa, lingocelulósico.

\section{Abstract}

This study presents the conceptual design of a pilot-scale downdraft fixed-bed gasifier of lignocellulosic biomass. The dimensions of the reactor were determined based on the requirement of electric power output $(150 \mathrm{kWt})$ and on the simulation of the gasification process with a one-dimension kinetic model. The simulations were aimed to obtaining high thermodynamic efficiencies (60\% and $75 \%$ ) for different biomass moisture contents. Depending on the reactor size and biomass moisture content, the simulations identified two best configurations for the gasifier that allowed generation of $117 \mathrm{kWt}$ and $200 \mathrm{kWt}$ with thermodynamic efficiencies of $43 \%$ and $70 \%$ respectively. The designed gasifier is $1,8 \mathrm{~m}$ long and produces between $25 \mathrm{kWe}$ and $50 \mathrm{kWe}$ as it makes use of the adiabatic characteristics of the endothermic zone and the gas sensitive enthalpy for biomass drying and devolatilization.

\section{Key words}

Downdraft gasifier, conceptual design, biomass, lignocellulosic biomass. 


\section{INTRODUCCIÓN}

Una de las tecnologías disponibles para el aprovechamiento energético de la biomasa sólida es la gasificación. En este proceso de transformación termoquímica se desarrolla combustión con defecto de oxígeno (inferior al estequiométrico), y su objetivo es transformar el combustible sólido en combustible gaseoso, denominado gas pobre, cuyo poder calorífico varía entre $4 \mathrm{MJ} / \mathrm{m}^{3}$ y $6 \mathrm{MJ} / \mathrm{m}^{3}$ para la gasificación con aire. El gas pobre se puede utilizar directamente en motores de combustión interna alternativos (MCIA), para producir energía mecánica o eléctrica mediante un generador (Lapuerta et al., 2004; 2007). Los gasificadores de lecho fijo en equicorriente (downdraft), son de uso común para la generación de bajas potencias eléctricas $(<500 \mathrm{kWe})$ debido a que producen un gas pobre más limpio (menor cantidad de alquitranes), aspecto que facilita el proceso de acondicionamiento y limpieza previa a su uso en MCIA. Este sistema de aprovechamiento energético de la biomasa permite emplearla en el mismo lugar en el que se genera y disminuir los costos derivados de su almacenamiento y transporte, los cuales son el principal reto al que se enfrenta el aprovechamiento energético de biomasa sólida (Melgar et al., 2008).

Pese a que diversas empresas en el mundo, principalmente en la India, Europa y Estados Unidos, han desarrollado la tecnología para gasificadores en lecho fijo equicorriente a nivel comercial, en la bibliografía publicada existe poca información en cuanto al diseño de gasificadores y sus sistemas auxiliares, que permita desarrollar sistemas propios de aprovechamiento energético. Sin embargo, Bacaicoa et al., presentan las dimensiones principales de dos gasificadores de lecho fijo en equicorriente (uno de $25 \mathrm{~kg} / \mathrm{h}$ a $50 \mathrm{~kg} / \mathrm{h}$ y otro de $200 \mathrm{~kg} / \mathrm{h}$ a $300 \mathrm{~kg} / \mathrm{h}$ ) (García-Bacaicoa et al., 1994) cuya tecnología data de la segunda guerra mundial, ya que en la zona de oxidación se construye una reducción del diámetro de garganta con el fin de craquear los alquitranes y transformarlos principalmente en metano, monóxido de carbono e hidrógeno. El gasificador de $50 \mathrm{~kg} / \mathrm{h}$ posee un diámetro variable entre $0,250 \mathrm{~m}$ 
y $0,5 \mathrm{~m}$, con una longitud de $1,73 \mathrm{~m}$, cuyo diámetro de garganta se diseñó con un criterio de $0,283 \mathrm{~kg} /\left(\mathrm{sm}^{2}\right.$ de garganta); mientras que el gasificador de $300 \mathrm{~kg} / \mathrm{h}$ posee un diámetro de $0,5 \mathrm{~m}$ a $1 \mathrm{~m}$ con una longitud de aproximadamente $2,8 \mathrm{~m}$, cuya garganta se diseñó cumpliendo el parámetro de $0,342 \mathrm{~kg} /\left(\mathrm{sm}^{2}\right.$ de garganta). Los materiales utilizados para la construcción de ambos reactores son aislamientos refractarios, cerámicos y acero. En la investigación experimental Bacaicoa et al. encontraron un rendimiento del proceso con el gas frío en torno al $70 \%$.

Tinaut et al. presentan el esquema de un gasificador de lecho fijo en equicorriente de una planta piloto de gasificación de 13 kWe (Tinaut et al., 2004.) el cual está construido por dos tubos concéntricos de acero al carbono. Estos mismos investigadores (Tinaut et al., 2004), presenta sin mucho detalle los esquemas de dos gasificadores, y una planta a nivel pre-industrial para generar $100 \mathrm{kWe}$. La planta consta de un gasificador de 4,5 m. de altura y $0,35 \mathrm{~m}$ de diámetro interior, sistema de limpieza y acondicionamiento del gas pobre, más cuatro motores de $2000 \mathrm{~cm}^{3}$ cada uno con sus respectivos generadores asíncronos.

Henriksen, et al., (2006) presentan una breve descripción de las condiciones de diseño, componentes y operación de una planta de generación de energía, la cual consta de un gasificador de dos etapas de lecho fijo en equicorriente. Esta planta operó por más de 465 horas continúas y permitió gasificar biomasa con un contenido de humedad del orden del $40 \%$ con un rendimiento global de la planta del $25 \%$. El gasificador se define de dos etapas, porque en una primera etapa mediante los gases de emisión del motor se promueve el secado y la devolatilización de la biomasa, mientras que en la segunda tiene lugar la oxidación parcial y la reducción, para dar lugar a la formación del gas pobre.

En la bibliografía consultada no se encontró ninguna descripción de cómo diseñar un gasificador de lecho fijo en equicorriente. En este trabajo se presenta una metodología de diseño conceptual de un gasificador de lecho fijo en equicorriente. El artículo describe los parámetros generales de operación del gasificador, sin entrar en detalle de descripción de las distintas partes. 


\section{REQUERIMIENTOS ENERGÉtICOS DEL GASIFICADOR}

Un gasificador debe cumplir ciertas especificaciones energéticas que, dentro del diseño conceptual, son necesarias al momento de evaluar las mejores alternativas de diseño. Se requiere también que el gas pobre, luego de pasar por los sistemas auxiliares, esté libre de alquitranes, material particulado y a la temperatura adecuada para ser utilizado como combustible en los MCIA. Este trabajo considera un reactor de lecho fijo equicorriente (downdraft) para gasificar biomasa lignocelulósica. Considera también que la biomasa utilizada como combustible debe tener una humedad menor o igual al 30\%, con el fin de obtener rendimientos con el gas frío en torno al $75 \%$.

El rango de potencia térmica generada debe estar entre 100 $\mathrm{kWt}$ y $200 \mathrm{kWt}$, valor típico si se quiere que el gasificador tenga aplicación en zonas interconectadas en Colombia, de acuerdo con el trabajo presentado por Tobón \& Agudelo; quienes desarrollan una herramienta multiobjetivo para evaluar la inversión en sistemas aislados sostenibles de energía (Tobón \& Agudelo, 2008). Los autores establecen que el consumo actual promedio de un habitante de una zona no interconectada a la red eléctrica nacional (ZNI) oscila entre 15 y $30 \mathrm{kWh} /($ mes-habitante). Asumiendo que la planta de generación de energía eléctrica opera por períodos de $18 \mathrm{hr} /$ día, por 25 días al mes, la potencia de diseño de la planta piloto de gasificación descrita en este trabajo $(25-50 \mathrm{kWe})$ tiene la capacidad de abastecer 750 personas en promedio, con una capacidad máxima a escala pre-industrial de abastecer 2450 personas, con un gasificador de mayores dimensiones acoplado a cuatro motores.

La generación distribuida en Colombia en las ZNI, mediante recursos energéticos renovables, supone una gran oportunidad para la ejecución de proyectos bajo la modalidad de Mecanismo de Desarrollo Limpio (MDL), brindando la oportunidad de participar en el mercado mundial con la venta de Reducción de Emisiones Certificadas (CER), aspectos que contribuirán a mejorar la calidad 
de vida de los habitantes de las ZNI, generando a su vez mayores posibilidades de inversión social desde la instancia gubernamental (Tobón \& Agudelo, 2008).

\section{Diseño conceptual del gasificador}

El diseño del gasificador toma como referencia la geometría general planteada en (Pérez, 2007) y que considera que el gasificador consta de una cámara de piro-combustión. Adicional al diseño geométrico del gasificador se incluye el de un intercambiador de calor de tubos concéntricos para aprovechar la energía sensible del gas pobre y favorecer los procesos de secado y devolatilización de la biomasa, ya que a medida que se incrementa la temperatura de entrada de los reactivos al proceso, se incrementa el rendimiento del mismo (Pérez, 2007).

La velocidad del proceso de reducción de la biomasa se regula mediante un sistema mecánico llamado parrilla que controla el tiempo de residencia de la fase sólida en el interior del gasificador, al remover la ceniza del fondo del mismo. Para el diseño de la parrilla y el intercambiador fue necesario evaluar varias opciones de diseño y se utilizó la metodología de Spinnler para seleccionar la mejor alternativa. (Spinnler, 1997).

\subsection{Dimensiones principales}

La siguiente secuencia se utilizó para definir los parámetros de diseño y la geometría preliminar del reactor:

- Determinar las dimensiones de la cámara de piro-combustión con base en experiencias reportadas en la bibliografía

- Probar la geometría del gasificador y verificar parámetros del proceso como rendimiento energético, poder calorífico del gas obtenido, mediante un modelo unidimensional del proceso de gasificación descrito en Pérez et al. 2007

La geometría de la cámara de piro-combustión consta de dos conos invertidos. Se considera que los procesos críticos durante la gasificación son la piro-combustión y la reducción del carbón 
vegetal, por lo que se toman como referencia para iniciar los cálculos de acuerdo con criterios ingenieriles, tal y como lo recomiendan Reed et al., (1994).

\subsection{Condiciones de operación}

\section{Consumo de combustible}

Reed et al., proponen un método de cálculo para el consumo de biomasa de acuerdo a la potencia requerida, el cual se ilustra en la Tabla 1 y se describe a continuación.

Tabla1. Cálculo del consumo de combustible EN FUNCIÓN DE LA POTENCIA REQUERIDA (REEd ET AL., 1994).

\begin{tabular}{lcc}
\hline \multicolumn{1}{c}{ Proceso } & Ecuación & Resultado \\
\hline Salida de potencia eléctrica & $\mathrm{EP}$ & $25-30 \mathrm{kWe}$ \\
\hline Rendimiento efectivo del motor & $\eta_{e p}$ & $25 \%$ \\
\hline Potencia térmica requerida del gas pobre & $E g=E p / \eta_{e p}$ & $200 \mathrm{~kW}$ \\
\hline Eficiencia del proceso de gasificación & $\eta_{e g}$ & $80 \%$ \\
\hline Poder calorífico de la biomasa (base seca) & $P C_{b m s}$ & $19565 \mathrm{~kJ} / \mathrm{kg}$ \\
\hline Consumo de Biomasa $\left(m_{b m s}\right)$ & $\mathrm{m}_{\mathrm{mbs}}=\mathrm{Eg} /\left(\eta_{e p}{ }^{*} P C_{b m s}\right)$ & $46 \mathrm{~kg} / \mathrm{h}$ \\
\hline
\end{tabular}

Tal y como se describió en el numeral 2 se fija una potencia eléctrica de entre $25 \mathrm{kWe}$ y $30 \mathrm{kWe}$ como parámetro de diseño del gasificador, con el fin de abastecer de energía eléctrica alrededor de 750 personas en promedio durante $18 \mathrm{~h} /$ día, por 25 días al mes.

El rendimiento efectivo de un motor de combustión interna alternativo de encendido provocado oscila entre $25 \%$ y $30 \%$ (Heywood, 1998). Para el diseño del gasificador se asume el límite inferior de tal rendimiento.

Como se discute a continuación, diversas publicaciones indican que el rendimiento energético en frío del proceso de gasificación se encuentra en torno al 75\%. (Zainal et al., 2002), estudian el 
efecto del poder calorífico y la tasa de producción de gas pobre al variar la relación combustible - aire en una planta de generación de energía eléctrica utilizando un gasificador de lecho fijo en equicorriente alimentado con residuos de madera, obteniendo rendimientos energéticos del proceso del 80\%, mientras que la eficiencia global del sistema produciendo energía eléctrica varía entre 10\% y 11\% con consumos específicos de biomasa de $2 \mathrm{~kg}$ $\mathrm{kW}^{-1} \mathrm{~h}^{-1}$. Warren et al. en una planta de similares características para la generación de energía eléctrica y térmica en equipos a pequeña escala, alcanza un rendimiento global del proceso del $20 \%$ con un consumo específico de combustible de $1 \mathrm{~kg} \mathrm{~kW} \mathrm{~kW}^{-1} \mathrm{~h}^{-1}$ (Warren et al., 1995). Además, Melgar, et al., (2007), mediante un estudio teórico-experimental del proceso de gasificación de biomasa en reactores de lecho fijo en equicorriente, encontraron que la biomasa debe poseer una humedad igual o inferior al 30\% en masa, para obtener rendimientos del proceso de gasificación en frío entre $80 \%$ y $85 \%$

\subsection{Dimensiones principales de la cámara de pirocombustión}

La cámara de pirocombustión, es el elemento fundamental del proceso de gasificación. Allí tiene lugar las reacciones exotérmicas que generan la energía para las reacciones endotérmicas; pirólisis, y reducción. A continuación se describen los principales elementos de este componente.

\section{Cono inferior de la cámara de piro-combustión}

Las dimensiones a calcular son diámetro mayor, diámetro menor y altura. Como hipótesis se plantea que en el interior del cono ocurren los procesos de secado, pirocombustión, y dos etapas de reducción. Estas dos últimas conforman el proceso de gasificación.

Para calcular la dimensión de la sección transversal de cada una de las regiones geométricas del cono se considera como variable de diseño la velocidad superficial del aire, pues ésta controla el consumo de biomasa por unidad de área y por ende la velocidad 
de avance del proceso (velocidad de avance del frente de llama). Finalmente, se calcula la longitud de las zonas con un método propuesto por (Pérez, 2007), donde se relacionan el tiempo de reacción para cada proceso y la velocidad de avance del mismo.

Tabla 2. Ecuaciones para el cálculo de las dimensiones del CONo inferior.

\begin{tabular}{|c|c|c|c|}
\hline Especificación & Criterio & $\begin{array}{l}\text { Ecuación } \\
\text { adaptada }\end{array}$ & Referencia \\
\hline $\begin{array}{l}\text { Cálculo del diámetro } \\
\text { mayor del cono }\end{array}$ & $\begin{array}{c}V S \geq 0,1 \mathrm{~m} / \mathrm{s} \\
m_{b m s}^{\prime \prime}=40 \cdot 10^{-3} \mathrm{~kg} /\left(\mathrm{m}^{2} \cdot \mathrm{s}\right)\end{array}$ & $m^{\prime \prime}{ }_{b m s}=\frac{m_{b m s}}{A_{1}}$ & (Pérez, 2007) \\
\hline $\begin{array}{l}\text { Cálculo del diámetro } \\
\text { menor de la zona de } \\
\text { piro-combustión }\end{array}$ & $\begin{array}{c}V S=0,15 \mathrm{~m} / \mathrm{s} \\
m_{b m s}^{\prime \prime}=40 \cdot 10^{-3} \mathrm{~kg} /\left(\mathrm{m}^{2} \cdot \mathrm{s}\right.\end{array}$ & $m{ }^{\prime \prime}{ }_{b m s}=\frac{m_{b m s}}{A_{1}}$ & (Pérez, 2007) \\
\hline $\begin{array}{l}\text { Longitud de la zona } \\
\text { de Piro-combustión } \\
\text { L1 }\end{array}$ & $\begin{array}{l}\text { Determinar la } \\
\text { longitud requerida } \\
\text { para llevar a } \\
\text { cabo el proceso de } \\
\text { piro-combustión }\end{array}$ & $\begin{aligned} L_{f p} & =V_{a p} \cdot t_{f p} \\
V_{a p} & =\frac{m_{b m s}}{A \cdot \rho_{b m s}}\end{aligned}$ & $\begin{array}{l}\text { (Reed et al., } \\
\text { 1994) }\end{array}$ \\
\hline $\begin{array}{l}\text { Tiempo de piro- } \\
\text { combustión } t_{f p}\end{array}$ & \multicolumn{2}{|c|}{$t_{f p}=\frac{0,207 \cdot \rho \cdot F s \cdot(1+1,76 \cdot F m) \cdot D \cdot(1+0,61 \cdot D) \cdot \exp (4369 / R T)}{\left(1+3,46 \cdot F_{O 2}\right)}$} & $\begin{array}{c}\text { (Reed et al., } \\
1994)\end{array}$ \\
\hline $\begin{array}{l}\text { Longitud para } \\
\text { reducción L2 }\end{array}$ & \multicolumn{2}{|c|}{ Ecuaciones (2),(3),(4) } & \\
\hline $\begin{array}{l}\text { Longitud de } \\
\text { remoción de cenizas } \\
\text { L3 }\end{array}$ & \multicolumn{2}{|c|}{$\begin{array}{c}\text { Se toma como criterio la recomendación } \\
\text { de } 18 \mathrm{~cm}\end{array}$} & $\begin{array}{c}\text { (García- } \\
\text { Bacaicoa et al., } \\
\text { 1994) } \\
\end{array}$ \\
\hline
\end{tabular}

La Tabla 2 resume el método de cálculo que permite estimar las dimensiones principales del gasificador y que considera la cámara de pirocombustión como elemento principal del reactor. De acuerdo con este método es posible encontrar que las dimensiones del cono inferior (ver figura 1) son D1=65 cm, D2=35 cm y L=30 cm. Para el cono superior se considera un diámetro mayor y uno menor de 65 $\mathrm{cm}$ y $25 \mathrm{~cm}$ respectivamente y con una altura de $40 \mathrm{~cm}$. 


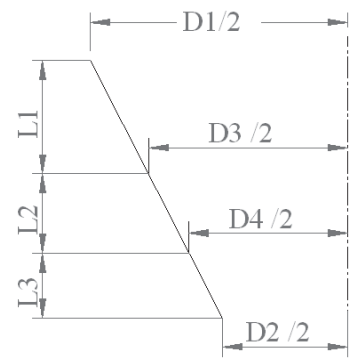

Figura 1. Dimensiones del CONO.

\section{Evaluación del diseño conceptual mediante una HERRAMIENTA COMPUTACIONAL Y ESTUDIO DEL EFECTO DE LA HUMEDAD DE LA BIOMASA EN EL PROCESO}

Con el fin de verificar el diseño conceptual desarrollado, en cuanto a potencia térmica y eficiencia, se utiliza un modelo dimensional para simular el gasificador, bajo diferentes condiciones de operación. Se estudia el efecto de la geometría del reactor y el contenido de humedad de la biomasa en el proceso termoquímico.

El modelo utilizado para determinar el efecto de la altura del gasificador y el contenido de humedad de la biomasa se basa en la integración de las ecuaciones conservativas con un planteamiento unidimensional y euleriano donde se caracterizan los procesos de secado, pirólisis y combustión/gasificación mediante la cinética química, teniendo en cuenta además los procesos de transmisión de calor (convección, conducción y radiación), fracción libre variable y disminución del tamaño de la partícula. La cinética química y el sistema de ecuaciones que conforman el modelo se describen de forma detallada en Pérez, (2007) y Tinaut et al, (2008).

La geometría encontrada en el numeral 3.2 se utiliza como entrada para el modelo (Pérez et al, 2007). Se consideraron como criterios para establecer la validez de la geometría los siguientes: 
(1) Potencia térmica $200 \mathrm{kWt}$, (2) eficiencia energética del proceso de gasificación entorno al 70\%, y (3) poder calorífico del gas pobre entre 4 - $6 \mathrm{MJ} / \mathrm{Nm}^{3}$ (Pérez, 2007).

\subsection{Parámetros simulados}

En el estudio paramétrico llevado a cabo para el diseño del gasificador se investigó el efecto de la geometría del reactor (variando la altura) y del contenido de humedad de la biomasa. Se eligen estos dos parámetros pues el costo de construcción del gasificador depende del tamaño del mismo y la potencia generada decrece considerablemente al aumentar el contenido de humedad de la biomasa. Se consideró que el contenido de humedad de la biomasa variará entre $10 \%$ y $30 \%$, pues si bien en la naturaleza la biomasa fresca se encuentra con porcentajes de humedad en torno al 50\% (Biofuelsb2b 2009; Meza et al., 2008), estos valores de humedad no proporcionan rendimientos aceptables del proceso, ya que demandan un gran consumo energético para evaporar el agua en el proceso, reduciendo así la temperatura de reacción y con ello la velocidad de gasificación. Sin embargo, cuando la biomasa se seca a la intemperie puede alcanzar porcentajes de humedad del $20 \%$, o incluso reducirse hasta un $10 \%$ mediante secado forzado (Meza et al., 2008).

En la tabla 3 se presentan las parámetros que se variaron en las cuatro simulaciones realizadas. La biomasa que se empleó en la simulación es madera de pino, cuya composición se presenta en la tabla 4 .

Tabla 3. Descripción de los cuatro casos estudiados en las simulaciones.

Simulación código Long. reactor (m) Humedad de la biomasa (\%)

\begin{tabular}{lll}
\hline S01 & 1,4 & 10 \\
\hline S02 & 1,4 & 30 \\
\hline S03 & 1,8 & 30 \\
\hline S04 & 1,8 & 10 \\
\hline
\end{tabular}


Tabla 4. Composición química de la madera de PINO.

\begin{tabular}{cc}
\hline Parámetro & Análisis elemental (\% b.s.) \\
\hline $\mathrm{C}$ & 52,48 \\
\hline $\mathrm{H}$ & 5,57 \\
\hline $\mathrm{O}$ & 40,95 \\
\hline Parámetro & Análisis inmediato (\% b.h.) \\
\hline Volátiles & 74,64 \\
\hline Carbono fijo & 14,30 \\
\hline Humedad & 10,00 \\
\hline Ceniza & 1,05 \\
\hline PCI (b.s.) & $19565 \mathrm{~kJ} / \mathrm{kg}$ \\
\hline
\end{tabular}

\subsection{Validación del diseño conceptual}

En la figura 2 se muestran los resultados de las cuatro simulaciones, las cuales son la base para determinar la altura final del gasificador, evaluando el efecto que tendrá el porcentaje de humedad de la biomasa en el proceso. En la figura 2 se observa que las mayores potencias se alcanzan con el gasificador de $1.8 \mathrm{~m}$ de altura. Con $10 \%$ de humedad en la biomasa es posible generar $200 \mathrm{kWt}$ y con un $30 \%$ se producen $118 \mathrm{kWt}$; mientras que con el gasificador de $1.4 \mathrm{~m}$ de longitud se generan entre $97 \mathrm{kWt}$ y 168 $\mathrm{kWt}$, estando el límite inferior ligeramente por debajo de los 100 $\mathrm{kWt}$, cuya potencia es el mínimo valor de diseño.

Una menor humedad en la biomasa genera una mayor potencia térmica debido a que disminuye la energía requerida para la evaporación del agua contenida en el combustible y aumenta la temperatura de gases, la velocidad de reacción y la cantidad de gases combustibles en la zona de reducción. De manera similar, pese a que el rendimiento energético, el dosado relativo de gasificación (figura 3a) y la composición del gas pobre (figura 3b) son muy similares se obtiene una mayor potencia térmica con el gasificador de mayor altura porque éste registra menos pérdidas de calor a través de las paredes, lo que aumenta la temperatura 
del gasificador y el flujo másico de las especies gaseosas. Con base en lo anterior, se seleccionó como longitud total del gasificador 1,8 m. Se decidió también que la cámara de pirocombsutión se construyera en cemento refractario, para favorecer el carácter adiabático del proceso.

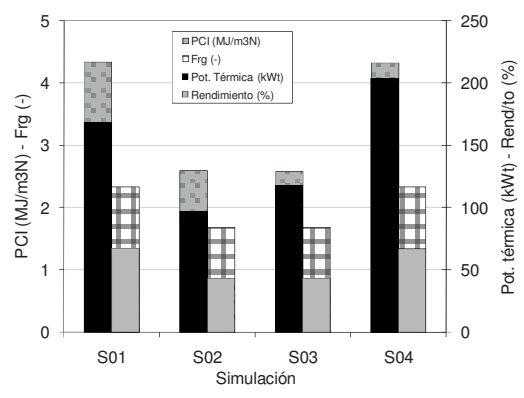

A) Parámetros característicos DEL PROCESO

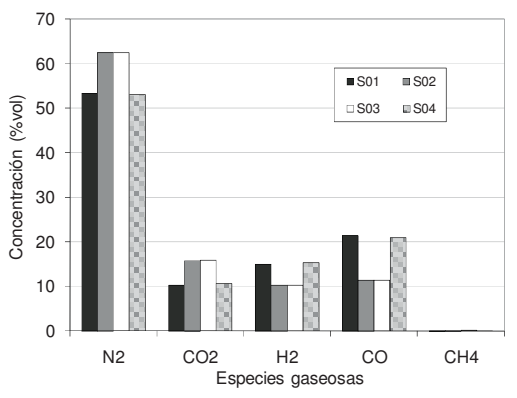

B) COMPOSICIÓN DEL GAS POBRE EN BASE SECA

Figura 2. Resultados de las simulaciones, eVAluANDO GeOMETRÍA del GasificAdor Y CONTENIDO DE HUMEDAD DE LA BIOMASA. LA TABLA 3 DESCRIBE EN DETALLE LOS PARÁMETROS QUE VARÍAN EN LAS SIMULACIONES

En la figura 3, se presenta, a lo largo del reactor, la concentración de las diferentes especies consideradas en el modelo, además de la distribución de las temperaturas tanto de la fase sólida, como la gaseosa en el gasificador. La figura permite identificar las principales zonas del proceso, y los puntos de mayor temperatura con el fin de definir los materiales para la construcción del reactor. Dada las temperaturas alcanzadas se debe elegir de forma adecuada el material de la cámara de pirocombustión, pues un acero al carbono puede transformase en austenita a los $800{ }^{\circ} \mathrm{C}$ y, dependiendo de la tasa de enfriamiento, se pueden obtener estructuras frágiles que se agrietan fácilmente, reduciendo el rendimiento al filtrase aire indeseado al proceso, y produciendo fallas mecánicas en la estructura si las grietas progresan. 


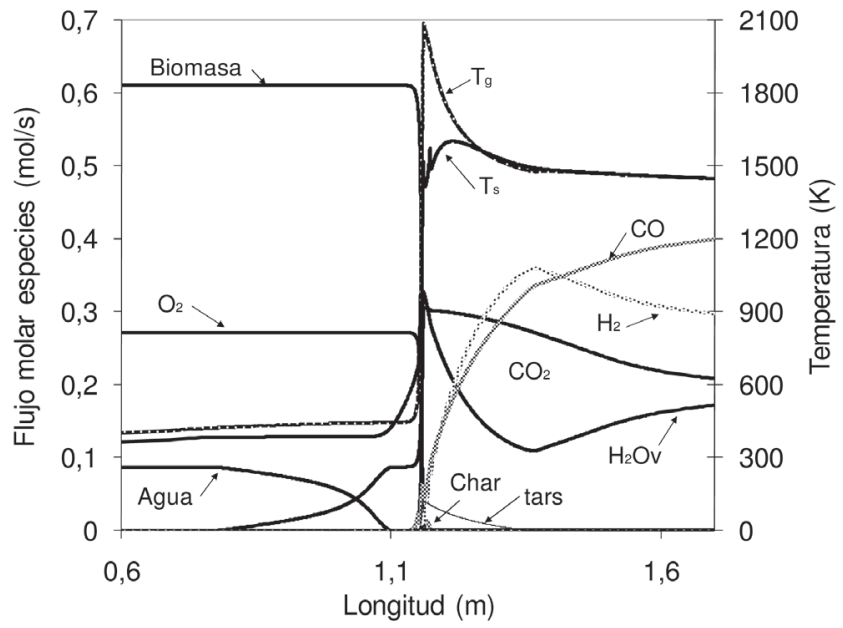

Figura 3. EVOLUCIÓN DE LAS ESPECIES Y LAS TEMPERATURAS ASOCIADAS AL PROCESO DE GASIFICACIÓN DE LA SIMULACIÓN S04.

El consumo específico de biomasa de la planta de generación de energía que utilice el gasificador diseñado oscilaría entre $0,9-1,6 \mathrm{~kg} \mathrm{~kW}_{\mathrm{e}}^{-1} \mathrm{~h}^{-1}$, con un rendimiento global entre 10 y $17 \%$.

Los valores energéticos por unidad de masa de la planta de generación de energía, tanto térmicos como eléctricos (ver tabla 5) coinciden con los valores reportados en la literatura (Biofuelsb2b, 2009).

TABla 5. ParÁmetros energéticos Por unidAd de MASA: PLANTA PILOTO VS. BIBLIOGRAFIA.

\begin{tabular}{ccccc}
\hline Tipo de biomasa & $\mathrm{kW}_{\mathrm{t}} \mathrm{h} / \mathrm{kg}$ & $\mathrm{kW}_{\mathrm{e}} \mathrm{h} / \mathrm{kg}$ & $\mathrm{MJ}_{\mathrm{t}} / \mathrm{kg}$ & $\mathrm{MJ}_{\mathrm{e}} / \mathrm{kg}$ \\
\hline Madera de pino $^{1}$ & $3,63-2,10$ & $0,91-0,53$ & $13,08-7,57$ & $3,27-1,89$ \\
\hline Trozos de madera $^{2}$ & 4,22 & -- & 15,2 & -- \\
\hline Residuos forestales $^{3}$ & 2,9 & -- & 10,51 & -- \\
\hline
\end{tabular}

$10-20 \%$ de humedad. Este trabajo.

$220 \%$ de humedad (Henriksen et al., 2006).

${ }^{3} 40 \%$ de humedad (Henriksen et al., 2006). 


\section{Diseño de OTROS COMPONENTES DEL GASIFICADOR}

La velocidad de la reacción de reducción del coque vegetal se controla mediante un sistema mecánico (parrilla) que regula el tiempo de residencia del carbón vegetal que reacciona con el dióxido de carbono, el vapor de agua y el hidrógeno productos de la devolatilización y la combustión.

Para el diseño de la parrilla se considera la alta temperatura (puede alcanzar temperatura de alrededor $800^{\circ} \mathrm{C}$, si no es refrigerada) a la cual está sometida y la demanda de movimiento de la misma. El diseño final después de evaluar varias alternativas, las cuales se describen en detalle en (Díaz \& Obando 2008), considera una parrilla de aletas con refrigeración a través del eje principal, como en la figura 4.

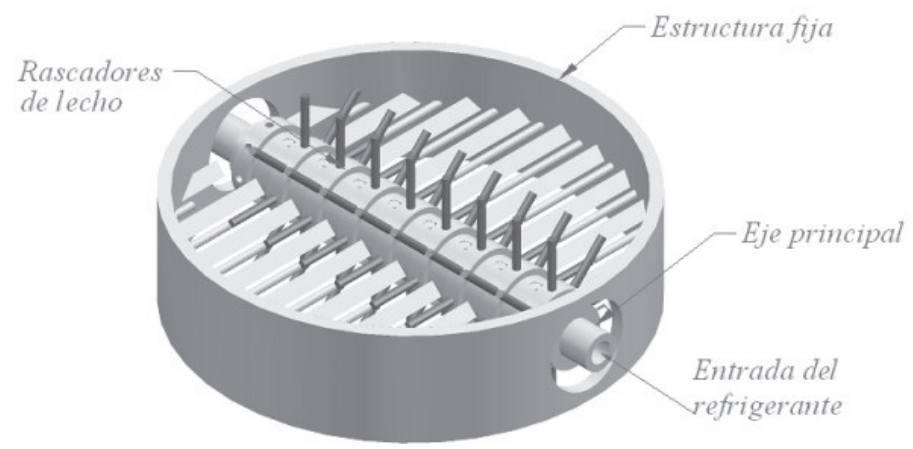

Figura 4. Diseño general de LA PARRILLA.

Un último elemento en el diseño del gasificador es un sistema de limpieza de gases que permita utilizar el gas pobre como combustible para MCIA según las especificaciones para contenido de alquitranes, material particulado y temperatura de entrada que se muestran en la tabla 6. Por tal razón, las plantas de generación de energía mediante gasificación de biomasa, deben estar equipadas con un sistema de limpieza y acondicionamiento del gas 
pobre, buscando una adecuada operación de la planta a la vez que minimiza los tiempos de parada por mantenimiento, figura 5

Tabla 6. Requerimientos del gas para ser alimentado en un MCiA (HAsler \& Nussbaumer, 1999).

\begin{tabular}{ccc}
\hline Requerimientos & Unidades & Valor \\
\hline Contenido de material particulado & $\mathrm{mg} / \mathrm{Nm}^{3}$ & $<50$ \\
\hline Tamaño de partícula & $\mu \mathrm{m}$ & $<10$ \\
\hline Contenido de alquitranes & $\mathrm{mg} / \mathrm{m}^{3} \mathrm{~N}$ & $<100$ \\
\hline
\end{tabular}

La temperatura de entrada del gas debe ser menor o igual a $50{ }^{\circ} \mathrm{C}$, con el fin de no reducir el rendimiento volumétrico del motor y con ello afectar la potencia mecánica, ya que éste es una máquina volumétrica y a mayor temperatura del gas disminuye su densidad, generando una menor cantidad de combustible por unidad de volumen, aspecto que afecta el rendimiento efectivo del motor. Con respecto al contenido de alquitranes hay diversas posiciones, sin embargo, la recomendación más frecuente es una concentración inferior a $100 \mathrm{mg} / \mathrm{m}^{3} \mathrm{~N}$. Para lograr los requerimientos de la tabla 6 se diseñó un sistema de acondicionamiento del gas que incluye una torre de lavado que se encarga de bajar la temperatura de $600^{\circ} \mathrm{C}$ a $100{ }^{\circ} \mathrm{C}$, retirar material particulado no depurado en un primer ciclón y condensar la mayor parte de alquitranes. Finalmente, el gas se hace pasar a través de dos lechos empacados que contienen biomasa igual a la utilizada en el gasificador, con el fin de reducir la humedad del gas pobre y atrapar alquitranes que hayan escapado de la torre lavadora. 


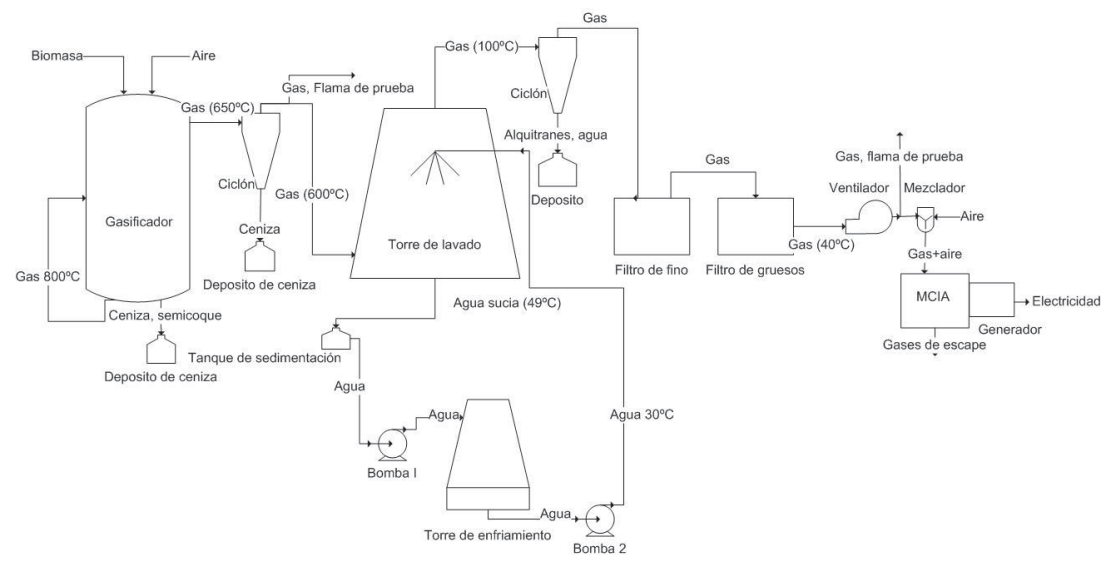

Figura 5. ESQUEMA DE GASIFICADOR DE BIOMASA CON SISTEMA DE LIMPIEZA Y ACONDICIONAMIENTO DE GAS POBRE PARA GENERACIÓN DE ENERGÍA ELÉCTRICA

\section{Conclusiones}

- De acuerdo con simulaciones realizadas para predecir el desempeño de un gasificador de biomasa de lecho fijo en equicorriente se encontró que al aumentar la longitud del gasificador, los cambios en el poder calorífico, dosado relativo y rendimiento del proceso, no fueron muy significativos, mientras que la potencia de salida y consumo de biomasa aumentaron, debido al incremento en el caudal del gas pobre, dando lugar a una mayor potencia térmica generada.

- Construir un gasificador de 1,8 m de longitud garantiza una potencia de salida de acuerdo con los requerimientos establecidos, además de una eficiencia térmica de gas frío del 67\%. El perfil de temperatura a lo largo del gasificador, muestra que las máximas temperaturas se encuentran a una altura entre $1,15 \mathrm{~m}$ y 1,8 m, lo que verifica que la cámara de piro-combustión debe tener una altura de $0,7 \mathrm{~m}$ igual a la establecida en un cálculo inicial. 
- El gradiente de temperatura máxima en una región del gasificador requiere de materiales que soporten térmicamente el proceso, por lo tanto el material para dichas condiciones debe ser refractario, dada su viabilidad económica frente a un acero inoxidable de baja conductividad térmica.

\section{Bibliografía}

Biofuelsb2b. (2009). Recuperado el 2007, de Typical calorific values of Biomass vs Fuel : http://www.biofuelsb2b.com/useful_info.php

Dasappa, S., Sridhar, H., Sridhar, G., Paul, P., \& Mukunda, H. (2003). Biomass gasifcation - a substitute to fossil fuel for heat application. Biomass and Bioenergy. Vol. 25. , 637-649.

Díaz, O. H., \& Obando, R. C. (2008). "Diseño de un gasificador de biomasa lignocelulósica a escala piloto", Trabajo dirigido de Grado para obtener titulo de Ingeniero Mecánico, Universidad Nacional de Colombia, Sede Medellín, Facultad de Minas. Medellín.

Garcia-Bacaicoa, P., Bilbao, R., Arauzo, J., \& Salvador, M. L. (1994). Scaleup of downdraft moving bed gasifiers $(25-300 \mathrm{~kg} / \mathrm{h})$ - design, experimental aspects and results. Bioresource Technology. Vol. 48, 229-235.

Hasler, P., \& Nussbaumer, T. (1999). Gas cleaning for IC engine applications from fixed bed biomass gasification. BIOMASS \& BIOENERGY , Vol 16 385-395.

Henriksen, U., Ahrenfeldt, J., Kvist, T., Gobel, B., Dall, J., Hindsgaul, C., y otros. (2006). The design, construction and operation of a $75 \mathrm{kw}$ twostage gasifier. Energy. Vol. 31, 1542-1553.

Heywood, J. (1998). Internal combustion engines fundamentals. First ed. New York: McGraw-Hill.

Lapuerta, M., Rodríguez, J., \& Hernández, J. (2007). Comparison between the kinetics of devolatilisation of forestry and agricultural wastes from the middle-south regions of Spain. Biomass and Bioenergy. Vol. 31, 1319.

Lapuerta, M., Rodríguez, J., \& Hernández, J. (2004). Kinetics of devolatilisation of forestry wastes from thermogravimetric analysis. Biomass and Bioenergy Vol. 27 , 385-391. 
Melgar, A., Borge, D., \& Pérez, J. F. (2008). Estudio cinético del proceso de devolatilización de biomasa lignocelulósica mediante análisis termogravimétrico para tamaños de partícula de 2 a $19 \mathrm{~mm}$. Dyna. Año 75 , 123-131.

Melgar, A., Pérez, J. F., Laget, H., \& Horillo, A. (2007). Thermochemical equilibrium modelling of a gasifying process. Energy Conversion and Management. Vol. 48, 59-67.

Meza, J., Gil, A., Cortés, C., \& González, A. (2008). Drying costs of woody biomass in a semi-industrial experimental rotary dryer. 16th European Conference \& Exhibition on Biomass for Energy, Biomass Resources. Valencia, Spain.

Pérez, J. F. (2007 ). Modelado unidimensional del proceso de gasificación de biomasa lignocelulósica en lechos empacados en equicorriente. Validación experimental con gasificadores invertidos. Valladolid, España: Tesis Doctoral, Universidad de Valladolid.

Pérez, J. F., Agudelo, J. R., \& Benjumea, P. (2007). Manual del usuario Emberá. Medellín, Colombia: Universidad de Antioquia, Universidad Nacional.

Reed, T. B., Graboski, M. S., \& Levie, B. (1994). Fundamentals, development \& scale-up of the air-oxygen stratified downdraft gasifier. Biomass Energy Foundation Press.

Spinnler, G. (1997). Introducción A Los Ejercicios De Diseño Y Construcción De Máquinas. En Capítulo 7 (pág. 49). Escuela Politécnica Ferederal de Lausana. Traducción y adaptación por: Jorge Arango. Universidad Nacional de Colombia sede Medellín.

Tinaut, F., Melgar, A., Horrillo, A., \& Diez, A. (2004.). Determination of the optimum parameters for a downdraft biomass gasifier through experimental design. World Conference and Technology Exhibition on Biomass for Energy, Industry and Climate Protection. Rome.

Tinaut, F., Melgar, A., Horrillo, A., \& Diez, A. (2004). Producción de energía eléctrica de origen renovable en pequeñas-medias potencias mediante gasificación de biomasa. XVI Congreso Nacional de Ingeniería Mecánica. León España.

Tinaut, F., Melgar, A., Pérez, J., \& Horrillo, A. (2008). Effect of biomass particle size and air superficial velocity on the gasification process in a downdraft fixed bed gasifier. An experimental and modelling study, . pp. Fuel Processing Technology. Vol. 89 , 1076-1089. 
Tobón, D., \& Agudelo, S. (2008). Optimización de herramientas multiobjetivo para la toma de decisiones de inversión en sistemas aislados sostenibles de energía. 1ra ed. Centro de investigaciones y consultorías - Universidad de Antioquia, 203-300.

Warren, T., Poulter, R., \& Parfitt, R. (1995). Converting biomass to electricity on a farm-sized scale using downdraft gasification and a spark-ignition engine. Bioresource Technology. Vol. 52, 95-98.

Zainal, Z., Rifau, A., Quadir, G., \& Seetharamu, K. (2002). Experimental investigation of a downdraft biomass gasifier. Biomass and Bioenergy. Vol. 23. , 283-289. 